\title{
Micro-structured electron accelerator for the mobile gas ionization sensor technology
}

\author{
C. M. Zimmer, K. T. Kallis, and F. J. Giebel \\ Intelligent Microsystems Institute, Faculty of Electrical Engineering and Information, Technische Universität \\ Dortmund, Dortmund, Germany \\ Correspondence to: C. M. Zimmer (cordula.zimmer@tu-dortmund.de)
}

Received: 31 October 2014 - Revised: 16 February 2015 - Accepted: 27 March 2015 - Published: 14 April 2015

\begin{abstract}
Mobile and economically priced gas monitoring and warning systems will become increasingly important for civil security, such as in fire brigade operations in undefined hazardous environments (Daum et al., 2006). Normally, photoionization detectors (PIDs) are used for the detection of gases. Hereby, the principle is based upon the ionization of the measured gas by photons, which are generated by a high-energetic gas discharge lamp with energy of 10-11 eV. Besides the detrimental unspecific gas detection because of the ionization of all gases with ionization potential (IP) below the provided photon energy, sensors also have a short lifetime combined with a high cost (http://www.intlsensor.com/pdf/photoionization.pdf).

This can be remedied by the concept of an electronic supported photoionization detector (ePID; Zimmer et al., 2012) consisting of a durable UV-LED with an above-positioned electron accelerator chip manufactured on a glass substrate by planar technology. Photoelectrons are extracted by UV illumination out of the bottom electrode and will be accelerated to an energy matching the ionization potential of the gas by a downstream acceleration grid. Thereby, the stable honeycomb-structured grid acts as a porous separator between the evacuated electron acceleration path due to $\mathrm{nm}$ scaling and the ionization area of the detector. To enhance the emitting area yielding a higher photoelectron current, the grid structure almost levitates, realized by the use of compatible planar technological processes such as reactive ion etching (RIE) and isotropic wet etching of sacrificial layers, which will be explained in detail in this paper. Furthermore, the tunability of the grid's acceleration voltage would enable a substance-specific determination of the gas composition, where the ionization of the analytes is clearly performed by photoelectrons instead of photons.
\end{abstract}

\section{Conventional possibilities and methods for gas detection}

Measuring instruments with different mechanisms are currently used for the detection of volatile gases, including a wide variety of potentially available chemical substances. Examples initially comprise the ion mobility spectrometer (IMS), the mass spectrometer (MS; Eiceman and Karpas, 2013), as well as Fourier transform infrared spectroscopy (FTIR). But, these measuring devices are partly suitable for on-site use. Alternative and portable measuring methods are presupposed by known analytes and limited by the TLV (threshold limit value) measurement done with the test tubes of the Dräger company. Another detection method is presented by the LIMS (laser ion mobility spectrometer), whereby substances are identified by their characteristic ionization potential detected by a tunable laser (Constapel et al., 2005). But, the expenditure for the realization of such a measuring system is tremendous. According to this, small and portable detecting systems such as conventional PIDs are increasingly in demand. All the above-mentioned methods are built up from discrete parts and are not suitable for realization as a single-chip system by planar technology. Lately, this is also reflected by the price of these systems. 


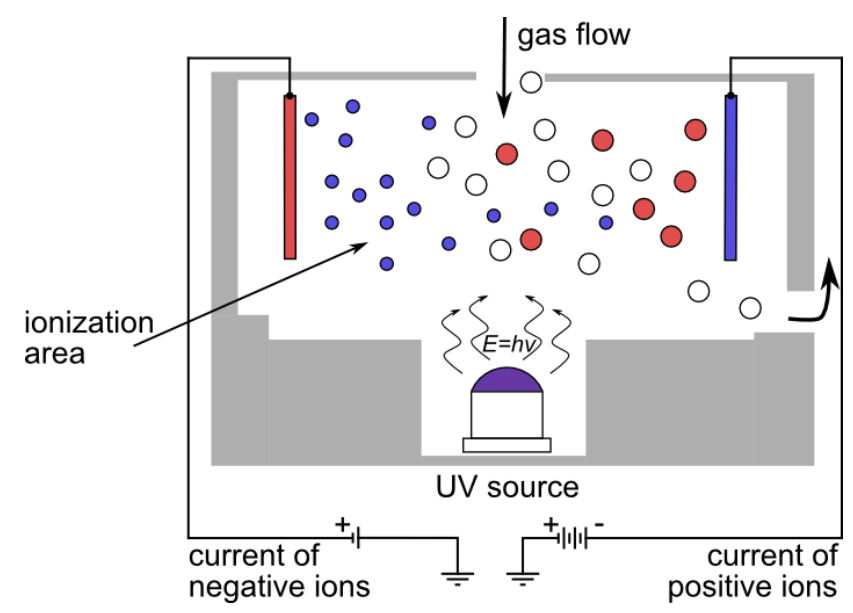

Figure 1. Basic principle of a conventional photoionization detector (PID).

\section{Gas sensor technology by photoionization detectors}

\subsection{Conventional photoionization detectors (PIDs)}

The structure of the conventional photoionization detector (PID) is shown in Fig. 1, and consists of an ionization chamber that includes a UV source and two capturing electrodes.

Hereby, the one-photon ionization based on the photoelectric effect is used by the PID for detecting the analytes. During ionization, the complete energy of the photon $(h v)$ is transferred to the gas atom $(B)$ as inelastic impact, whereupon the gas atom $\left(B^{+}\right)$is ionized by extracting an electron $\left(e^{-}\right.$; Eiceman and Karpas, 2013).

$B+h v \stackrel{\text { Ionization }}{\longrightarrow} B^{+}+e^{-}$

In case of gas ionization by a photon, the provided photon energy has to be larger than the ionization potential (IP) of the atom or molecule ( $h v>$ IP). According to this, substances in the air such as aromatic hydrocarbons and organic compounds are detected by the PID in terms of their ionization potentials. Thereby, substances are introduced into the ionization area of the detector by a pump or easily by diffusion. Ionized atoms or molecules resulting from impacts by photons and free electrons are attracted by the biased electrodes, respectively. Afterwards, a discharge and current flow accordingly occurs, which is converted into a signal by processing electronics. The higher the available substance concentration, the higher the detectable current. Hence, systemspecific parameters like the dimension of measurement cells as well as biased voltage at electrodes have to be considered and calibrated on the particular gas for converting into the parts-per-million range. Typically, a gas discharge lamp filled with inert krypton gas for generating photon energy of $10.6 \mathrm{eV}$ is used, whose energy is high enough to ion- ize volatile organic compounds (VOCs) without ionizing the so-called background molecules such as oxygen, nitrogen and water vapor that have an ionization potential of over $10.6 \mathrm{eV}$. Therefore, the ionization of substances is not only dependent on the introduced energy, but also on the cross section of ionization (http://www.intlsensor.com/pdf/ photoionization.pdf, Eiceman and Karpas, 2013, Freedman, 1980). Only a certain part of the molecule structure is ionized and registered by the PID, which is dependent on substance specification but not on substance concentration. For that reason, so-called response factors are necessary, which are detected as a reference to a gas like 2-methylpropene. These response factors are determined in time-consuming experiments, but are indispensable to making a relevant statement about the ionization probability.

\subsection{Disadvantages of photoionization detectors (PIDs)}

Unfortunately, the PID cannot exactly identify lightly or heavily ionizable substances in gas compounds, which are introduced in the measurement cell. It is also difficult to make a statement about its concentration. Lately, a PID can only determine the amount of ionized gaseous substances (Constapel et al., 2005)

During the use of PIDs, some mistakes took place, resulting from different factors and gaining more and more relevance with increasing operation time. One of the key factors is the influence of dust and humidity and the degradation of components, which cannot be corrected by processing electronics.

Before using the PID, a reference routine is made, where the test gas, usually 2-methylpropene with a known concentration, is pumped into the PID. The 2-methylpropene is ionized without any influences and its concentration is displayed. Normally the test gas is a dry gas, but unfortunately air humidity is present during on-site usage under ambient air, and ionizations can be hindered.

This is a result of water attraction on formed ions or electrons decreasing the mobility and ionizing capabilities (quench effect; RAE Systems Inc., 2013). With this effect, an offset of $20-30 \%$ to the reference value occurs. To minimize the influences of air humidity, a humidity sensor is installed in the PID, separately determining the air humidity and contributing it to the measuring process (http://www.intlsensor. com/pdf/photoionization.pdf, RAE Systems Inc., 2013).

Other influences on measuring signals are equally performed by contaminations in the air, meaning electrodes are contaminated by dust particles penetrating into the measuring cell, causing a capillary condensation. Due to existing salts in the dust layer, a current flow proceeds. This effect is called parasitical conductivity and will be substantially enhanced by the humidity in ambient air.

Preventing the penetration of dust particles, the PID is equipped with dust filters, which are cleaned up or replaced at periodic time intervals to avoid measuring errors (RAE 


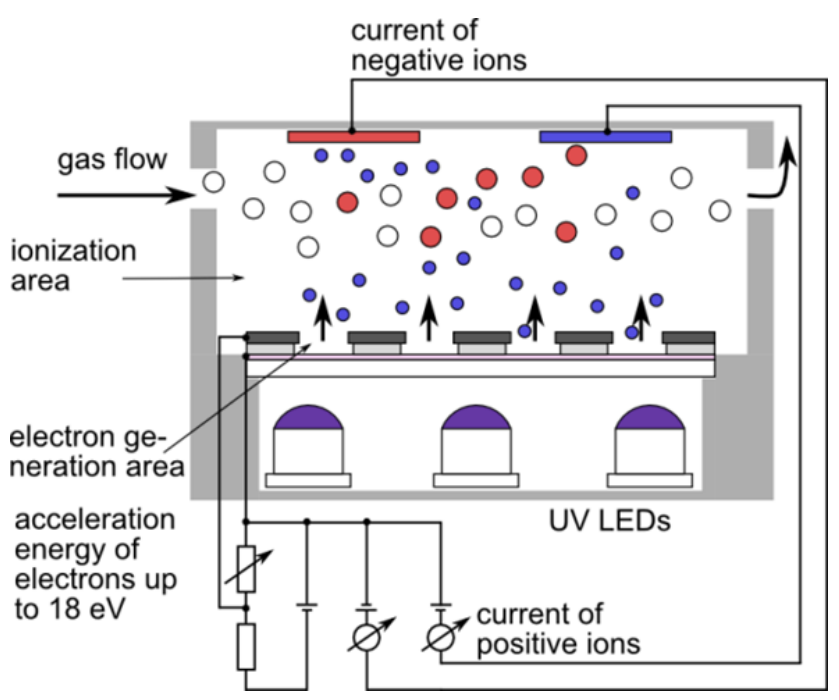

Figure 2. Basic principle of an electronic supported photoionization detector (ePID; Zimmer et al., 2012).

Systems Inc., 2013). Another negative influence on the detection by PID is represented by bulb degradation of the UV light source. Here, the glass bulb of the UV lamp consisting of magnesium oxide or magnesium fluoride is roughened by the extraction of high-energetic photons. This damage supports the reflection of photons at the glass, leading to a complete blindness of the UV light source, performing no further gas ionizations (Zimmer et al., 2012).

\subsection{Electronic supported photoionization detector (ePID)}

Not all the above-mentioned disadvantages of the PID can be compensated for the use of the electronic supported photoionization detector (ePID) presented here. Besides the quench effect, the main problems such as high maintenance costs due to the replacement of a degenerated discharge lamp as well as the imprecise detection of volatile organic substances can be solved by using an optimized detector via a micro-structured accelerator. The ePID works on the same principle as a PID. The substances to be detected in air are aspirated and ionized by the detector. These gases are measured as an ionization current due to biased electrodes and registered, analyzed and displayed by the processing electronics. The most significant difference between the ePID and the PID explained above is the type of gas ionization. Instead of a direct ionization by high-energetic photons, an indirect ionization by photoelectrons with the help of an electron emitter being subjected to the photoelectric effect and a downstream honeycomb-structured acceleration path fabricated by planar technology is performed. The system of such an ePID is schematically shown in Fig. 2.

Hereby, the detrimental rapid degradation of the UV source due to radiation damage is prevented by this modi- fication of the PID. And the use of an expensive and highenergetic UV lamp becomes invalid. The electron emitter consisting of lanthanum hexaboride $\left(\mathrm{LaB}_{6}\right)$ exhibits a low crystal-dependent work function of $2.3 \mathrm{eV}$ (Nishitani et al., 1980). As a primary photon source, a comparatively inexpensive UV-LED is chosen whose wavelength is matched to the work function of the emissive layer. Due to the illumination, photoelectrons are extracted from the emitter that has a kinetic energy of almost $0 \mathrm{eV}$ (Zimmer et al., 2012) due to optimum adjustment of the light source and emissive layer, meaning the photoelectrons only perform disordered thermal movements.

Afterwards, photoelectrons are accelerated by a downstream acceleration path towards the ionization area to perform the gas ionization. Due to the application of different voltages at the acceleration grid, a wide range of electron energies can be realized. Besides a better selectivity, ionization energies above the typical $10.6 \mathrm{eV}$ from commercial PIDs can also be implemented. But, to preserve the measurement of significant results, an acceleration energy of $25 \mathrm{eV}$ should not be exceeded because of the formation of fragment ions.

Hereby, the geometric expansion of this acceleration path is another important aspect, which should be smaller than the mean free path of electrons in air. Thus, it is guaranteed that the use of expensive vacuum components can be waived. Furthermore, it is not advantageous to position the UV-LED directly into the ionization area because of the indirect gas ionization. Therefore, a backside illumination through a UV transmissible substrate such as borosilicate glass (BOROFLOAT ${ }^{\circledR} 33$ ) is considered to benefit the noninteraction between photons generated by UV-LED and gas molecules in the ionization area, gaining a higher yield of photoelectrons from the emitter (Zimmer et al., 2012). This means that, besides the lanthanum hexaboride, another material has to be chosen as the counter-electrode to the acceleration grid, having high UV transmission behavior and good electrical properties. For this purpose, indium tin oxide (ITO) is a suitable candidate known from the display and solar cell industry.

\section{Fabrication of ePIDs by planar technology}

\subsection{Process flow}

The process of an ePID chip starts with the deposition of an indium tin oxide layer on borosilicate glass by the magnetron sputtering technique. Then, lanthanum hexaboride $\left(\mathrm{LaB}_{6}\right)$ is sputtered as the emitter layer on top of it. The deposition of an insulating layer of silicon dioxide in a PECVD (plasma enhanced chemical vapor deposition) reactor is followed using silane thinned by argon $\left(\mathrm{SiH}_{4} / \mathrm{Ar}\right)$ and dinitrogen monoxide $\left(\mathrm{N}_{2} \mathrm{O}\right)$ as processing gases. The front electrode consists of titanium nitride (TiN), a mechanically stable material. Here, the magnetron sputtering technique is also performed to gain a high layer quality of TiN. Hence, the deposition of tita- 


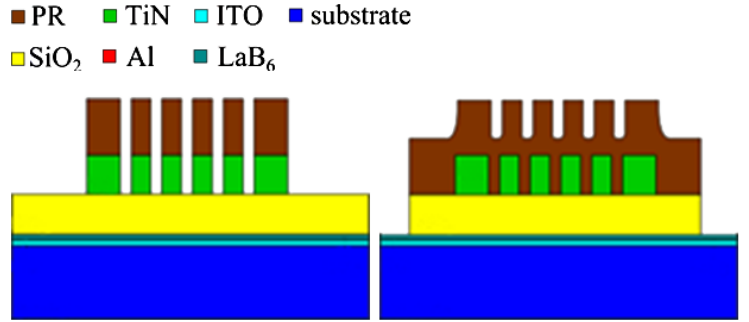

Figure 3. Schematic cross section of the detector chip after the patterning of a front electrode (left) and after etching the mesa (right) (Kallis et al., 2014).
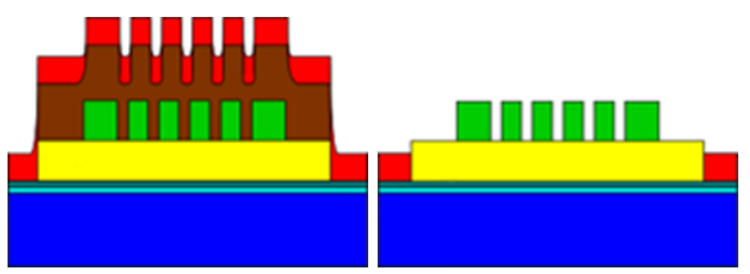

Figure 4. Cross section of the detector chip before (left) and after (right) the lift-off process of aluminum contacts (Kallis et al., 2014).

nium is reactively executed with the addition of nitrogen. Then, this front electrode is anisotropically etched by means of conventional photo lithography in a parallel plate reactor. The appropriate processing gases are nitrogen $\left(\mathrm{N}_{2}\right)$, chlorine $\left(\mathrm{Cl}_{2}\right)$, silicon tetrachloride $\left(\mathrm{SiCl}_{2}\right)$ and methane $\left(\mathrm{CH}_{4}\right)$ for the sidewall passivation. The cross section of the sensor chip after the patterning of the front electrode is shown in Fig. 3.

Afterwards, an anisotropic etching step forming a mesa structure took place to isolate the chips from each other and contact the bottom electrode (Fig. 3, right). This step is also performed by a parallel plate reactor of a reactive ion etching system using trifluormethan $\left(\mathrm{CHF}_{3}\right)$ and argon $(\mathrm{Ar})$ as process gases. The contact with the bottom electrode including lanthanum hexaboride and indium tin oxide, respectively, is realized by lift-off structured aluminum. The cross section of the detector chip before (left) and after (right) the removal of photoresist is shown in Fig. 4.

In the following, a deposition of another PECVD silicon dioxide layer is performed as a passivation film for the aluminum metal circuit paths. As a next step, the front electrode has to be levitated with the help of buffered hydrofluoric acid (BHF) (cf. Fig. 5, right). To protect the passivating oxide and circuit paths against the etching procedure, a resist mask is used during the wet chemical etching (cf. Fig. 5, left).

The process is concluded by exposing the connections of the still fully passivated bottom electrode due to contact holes. A cross section of the complete processed detector chip is shown right in Fig. 6.
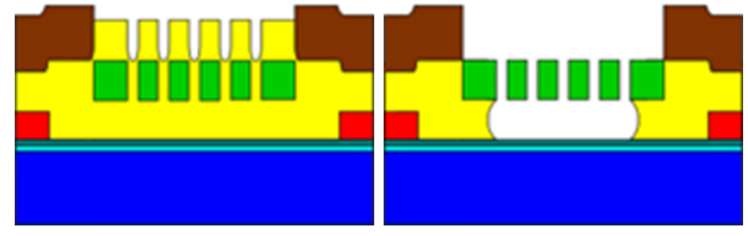

Figure 5. Cross section of the detector chip before (left) and after (right) wet chemical etching of silicon dioxide (Kallis et al., 2014).
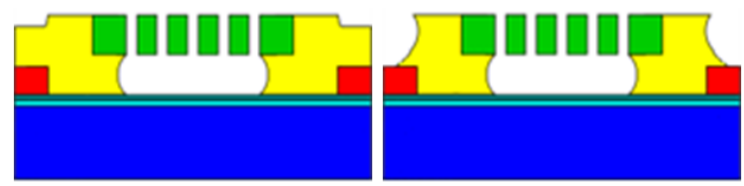

Figure 6. Cross section of the detector chip before (left) and after (right) the exposure of contact holes (Kallis et al., 2014).

\subsection{Dimensioning and boundary conditions}

In addition to modeling of the front electrode, the optimization of deposited thin films' quality and their dimensioning is also of importance.

\subsubsection{Bottom electrode}

First, the ITO layer on the glass substrate has to be optimized in terms of its transmission and conductivity. The transmission of ITO in relation to the used wavelength as well as deposited film thickness is shown in Fig. 7. A better transmission is obviously recognizable in thin films for the deep ultraviolet wavelength range.

Furthermore, an increase in transmission is shown with increasing film thickness at $50 \mathrm{~nm}$ at a wavelength of approx. $365 \mathrm{~nm}$, which is explainable due to the interference effects at the interface between the substrate and the ITO layer (Zimmer et al., 2013). In accordance with the adjustment of the UV source and the emissive layer, an optimum between transmission and conduction behavior of ITO can be achieved.

\subsubsection{Photoelectron emitter}

The following layer used as the electron emitter has to be deposited very thinly, guaranteeing the escape of photoelectrons from the front side by backside UV illumination. It is also important to consider that the used lanthanum hexaboride $\left(\mathrm{LaB}_{6}\right)$ as emitter material is not totally inert in normal environments, resulting in a slight oxidation of the surface, because adsorbates are collected on the surface during the operation of the ePID chip, inducing the increase in the electron's work function of up to $1 \mathrm{eV}$ (Nishitani et al., 1980). Besides these degenerative effects, the work function is also influenced by the crystallinity of the thin film. But, with an illumination of the emitter layer by a wavelength of $365 \mathrm{~nm}$, 


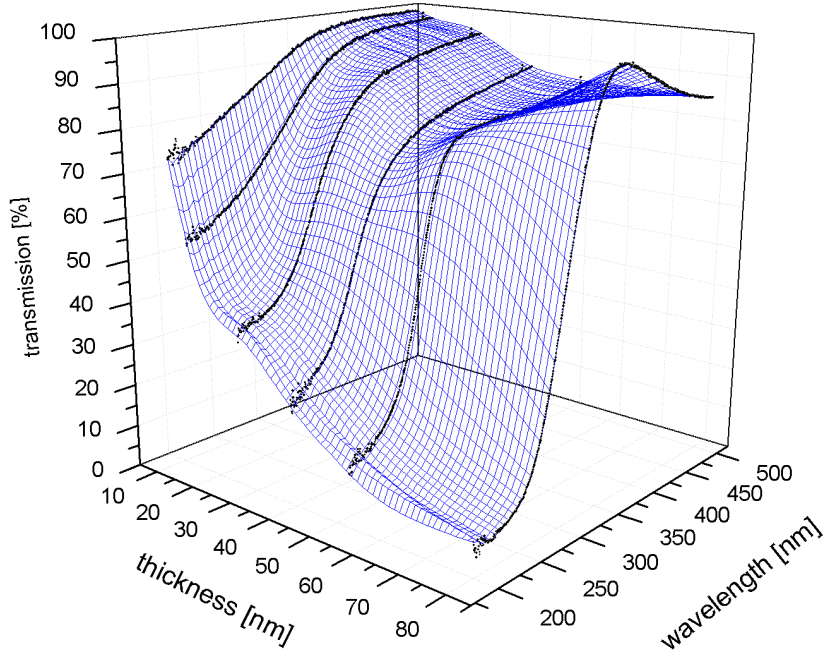

Figure 7. Transmission of the bottom electrode ITO in relation to film thickness and used wavelength (Zimmer et al., 2013).

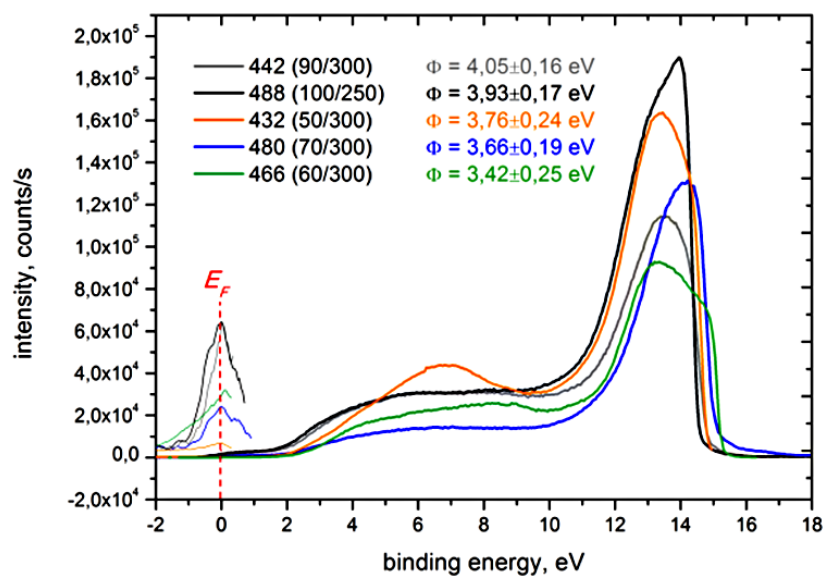

Figure 8. Ultraviolet photoelectron spectra of analyzed $\mathrm{LaB}_{6}$ films (including sample numbers and deposition parameters; argon flow/DC power) with corresponding measured work functions (Zimmer et al., 2014).

the work function should be lower than $3.39 \mathrm{eV}$. Although work function values below $2.5 \mathrm{eV}$ are specified in the literature (Yamauchi et al., 1976), higher values are presented by both evaporated and sputtered as well as PLD (pulsed laser deposition) deposited layers after storage in air (Zimmer et al., 2013). In this work, $\mathrm{LaB}_{6}$ thin films are deposited by the magnetron sputtering technique using DC power of $300 \mathrm{~W}$ and a processing argon flow of $60 \mathrm{sccm}$. Thereby, as a result, a (100) crystalline texture is reached, benefiting the work function of the material (Yamauchi et al., 1976). Due to decreasing argon flow rate at constant DC power, a reduction in the work function can be seen in Fig. 8, forwarding a higher atomic ratio of boron to lanthanum $(\geq 6)$ and decreasing the internal stress (Zimmer et al., 2014).

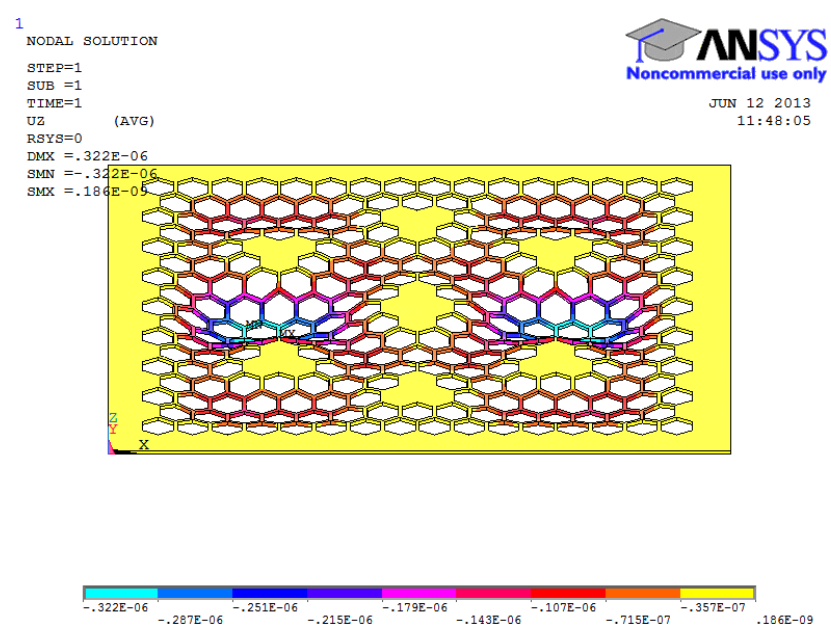

Figure 9. Deformation of a TiN grid structure with supporting elements by maximum electrostatic stress.

On the other hand, a change to other materials is possible, such as samarium oxide (Constantinescu et al., 2012). But, experiences concerning long-term stability and high-quality film depositions are currently still missing.

\subsubsection{Insulator}

The following deposited silicon dioxide film is used as the insulation layer for the front electrode. In particular, the optimized PECVD oxides are ideally suited for this purpose, because of their dielectric strength in the range of $100 \mathrm{mV} \mathrm{nm}^{-1}$. But, for sufficient dimensioning of this area, the mechanical deformation of the front electrode is also a decisive factor, as well as the dielectric strength. Since the acceleration path is used in air, a maximum layer thickness of $500 \mathrm{~nm}$ must not be exceeded, which corresponds to the free mean path of electrons in air (Seah and Dench, 1979). The insulator is also used as a buffer layer against the mechanical stress that occurred between the ITO and the TiN electrode. The deposition of $\mathrm{SiO}_{2}$ is performed by a combination of high- and low-frequency plasma excitation to decrease the intrinsic layer tension at greater film thicknesses (Tarraf et al., 2004).

\subsubsection{Front electrode}

From the mechanical point of view, the front electrode is the most stressed part of the detector chip. To gain the highest electron yield possible, the grid has to be realized as almost self-supporting. Furthermore, it has to carry its own weight completely and has to compensate for the electrostatic forces due to the applied acceleration voltage. For this purpose, a honeycombed grid structure with supporting elements of silicon dioxide is used, which is optimized with the help of the finite element method (see Fig. 9, Kallis et al., 2014). 

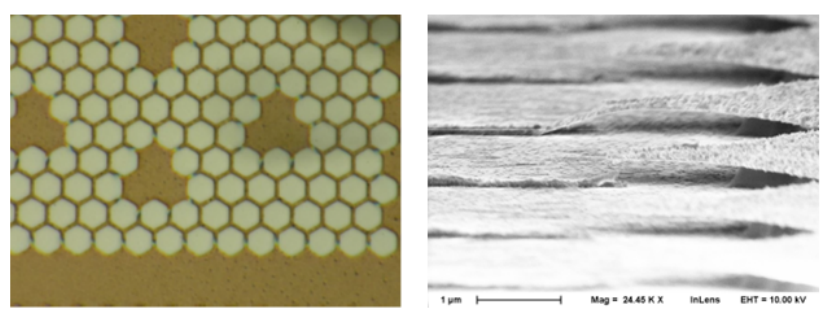

Figure 10. Image of a collapsed ePID chip taken by microscope (left) and scanning electron microscope (right).
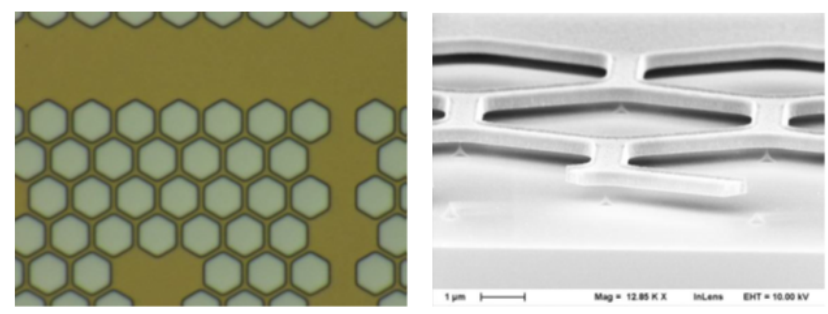

Figure 11. Image of an optimized ePID chip taken by microscope (left) and scanning electron microscope (right, Kallis et al., 2014).

However, the biggest challenge of the front electrode is described by its fabrication considering the operation conditions. Due to an incorrect dimensioning or the use of unsuitable materials, the grid structure can be collapsed irreversibly either during fabrication or operation by applying acceleration voltage. Thereby, an optimum adjustment between the grid thickness and the applied voltage has to be made.

By applying excessive voltages, the grid structure collapses, which is shown exemplarily in Fig. 10. Here, strong deformations are already divined by the microscope (left) based on the irregular reflection behavior of the front electrode. This assumption is confirmed by the oblique view of the chip surface taken by a scanning electron microscope (SEM). Obviously, a short-circuit is performed due to adhesion of the front electrode on the lower electrode by leaving the area of reversible elastic deformation. Besides the exact geometric dimensioning, the use of complete stoichiometric titanium nitride is essential because of its optimal mechanical stiffness, preventing the collapses of the front electrode. The image of a perfect ePID chip taken by both a microscope (left) and a scanning electron microscope (right) is shown exemplarily in Fig. 11. The almost levitating grid is only stabilized by its frame structure and the supporting points, where no deformation occurred.

\section{Discussion}

The processes presented in the previous sections were investigated with the help of electrical and mechanical simulations at the Technology of Research and Application Development of TU Dortmund, and required that processes were optimized

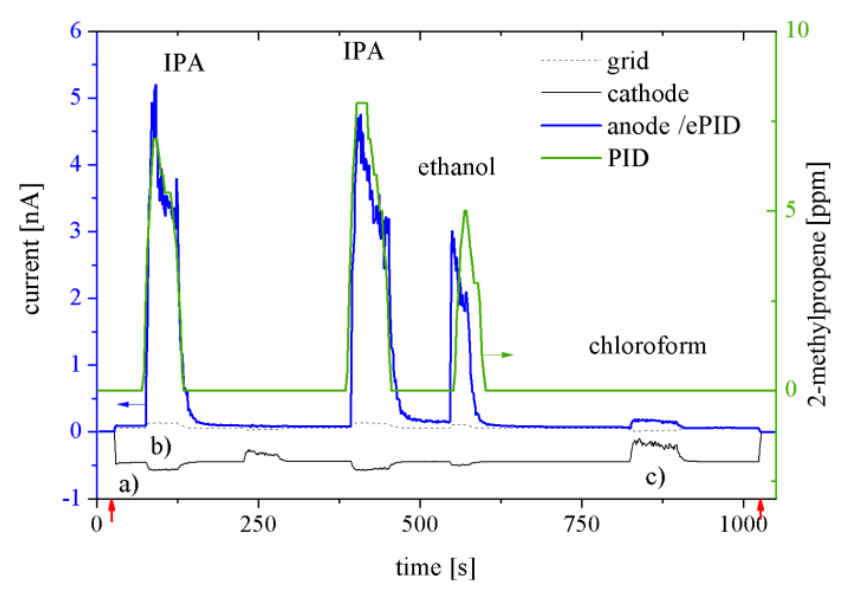

Figure 12. Measured ionization signal of PID calibrated to 2methylpropene (green signal) and compared to an ePID (blue signal). (a) Emission of photoelectrons out of the cathode, (b) ionization by photons, and (c) ionization by emitted photoelectrons (Zimmer et al., 2012).

continuously. Hereby, the technical feasibility as well as the superiority of such a detector is already presented by given prototypes (Zimmer et al., 2012).

First, the ionization behavior of the ePID is registered and compared to the PID used as the reference signal. During ionization measurement, the same gas discharge lamp with a wavelength of $116 \mathrm{~nm}$ is used as the UV source to guarantee the photoelectric effect in the ePID. The detected signal of evaporated solvents by both detectors is shown in Fig. 12 . Hereby, the majority of gaseous substances is detected by an ePID (see the blue signal in Fig. 12), having a downstream acceleration path instead of a classical PID.

Both 2-propanol (IPA, $\left.\mathrm{C}_{3} \mathrm{H}_{7} \mathrm{OH}\right)$ and ethanol $\left(\mathrm{C}_{2} \mathrm{H}_{5} \mathrm{OH}\right)$ with ionization potentials of 10.15 and $10.5 \mathrm{eV}$, respectively, are detected by PID and ePID due to the dominating ionization process by photons. But, only the ePID is able to deliver a signal by introducing evaporated chloroform $\left(\mathrm{CHCl}_{3}\right)$ into the measuring cell, which has an ionization potential of $11.4 \mathrm{eV}$, exceeding the used photon energy of $10.6 \mathrm{eV}$ (Zimmer et al., 2012). Here, only photoelectrons emitted by the gas discharge lamp $(10.6 \mathrm{eV})$ ionize chloroform molecules shown as a signal in Fig. 12c. The ionization behavior of the ePID is also successfully performed by the use of a UV-LED $(\lambda=260 \mathrm{~nm})$ as a radiation source (Zimmer et al., 2012). But, the achieved signal strength is still too low and has to be improved by further optimization of used masks and materials according to Sect. 3.2. Thus, additional investigations have to be done, particularly concerning the dependence of gas selectivity on the variation of acceleration voltage. Presumably, there is an offset between the applied grid voltage and the gas' ionization potential due to existing contaminations on diverse surfaces performing uncontrollable ionization effects. In particular, the possible formation of ions 
caused by clustering with water molecules has to be analyzed in detail by spectrometric methods.

\section{Conclusion and outlook}

In this paper, the basics for the dimensioning and fabrication of novel and innovative electronic supported photoionization detectors (ePID) has been presented.

Here, ePIDs point out further advantages, including the enormous cost reduction due to the application of planar technological processes known from chip manufacture compared to already existing sensors. A wide spectrum of application fields is especially feasible due to a higher selectivity by the tunability of the sensor chip as well as the expanded ionization range by a factor of 2 compared with UV lamps. Despite the proven basic functionality of an ePID on a laboratory scale, a long way remains for optimization and further development until its realization in a finished product, in detail, mainly the optimization of the electronic work function of the emissive layer regarding the long durability for the possible use of low-cost UV-LEDs as light sources. Another important aspect in further investigations is the additional integration of capturing electrodes into the chip, which still have to be built up from discrete parts.

Acknowledgements. The authors would like to thank Karola Kolander, Marko Kremer and Achim Wiggershaus for the technical support with the manufacture of prototypes.

In addition, the persons concerned would like to thank the Federal Ministry of Education and Research for the financial support of the study.

Edited by: J. Zosel

Reviewed by: two anonymous referees

\section{References}

Constantinescu, C., Ion, V., Galca, A. C., and Dinescu, M.: Morphological, optical and electrical properties of samarium oxide thin films, Thin Solid Films, 520, 6393-6397, 2012.

Constapel, M., Schellenträger, M., Schmitz, O. J., Gäb, S., Brockmann, K. J., Giese, R., and Benter, Th.: Atmospheric-pressure laser ionization: A novel ionization method for liquid chromatography/mass spectrometry, Rapid Commun. Mass Sp., 19, 326336, 2005 .

Daum, K. A., Watrous, M. G., and Neptune, M. D.: Data for First Responder Use of Photoionization Detectors for Vapor chemical Constituents, Idaho National Laboratory, Idaho Falls, Idaho, USA, 2006.

Eiceman, G. A. and Karpas, Z.: Ion Mobility Spectrometry, 3rd Edn., Taylor \& Francis Group, New York, USA, 2013.

Freedman, A. N.: The photoionization detector: Theory, performance and application as a low-level monitor of oil vapour, J Chromatogr. A, 190, 263-273, 1980.

Kallis, K. T., Dietz, D., Subasi, E., Müller, M. R., Kontis, C., and Zimmer, C. M.: Design, simulation, fabrication and characterization of nano-scaled acceleration grids, Microelectronic Engineering, 121, 118-121, 2014.

Nishitani, R., Aono, M., and Tanaka, T.: Surface structures and work functions of the $\mathrm{LaB}_{6}$ (100), (110) and (111) clean surfaces, Surf. Sci., 93, 535-549, 1980.

RAE Systems Inc.: The PID Handbook: Theory and applications of direct reading photoionization detectors (3rd Edn.), ISBN 09768162-1-0, 2013.

Seah, M. P. and Dench, W. A.: Quantitative electron spectroscopy of surfaces: A standard data base for electron inelastic mean free paths in solids, Surf. Interf. Anal., 1, 2-11, 1979.

Tarraf, A., Daleiden, J., Irmer, S., Prasai, D., and Hillmer, H.: Stress investigation of PECVD dielectric layers for advanced optical MEMS, J. Micromech. Microeng., 14, 317-323, 2004.

Yamauchi, H., Takagi, K., Yuito, I., and Kawabe, U.: Work function of $\mathrm{LaB}_{6}$, Appl. Phys. Lett., 29, 638, 1976.

Zimmer, C. M., Kieschnick, M., Kallis, K. T., Schubert, J., Kunze, U., and Doll, T.: Nano photoelectron ioniser chip using $\mathrm{LaB}_{6}$ for ambient pressure trace gas detection, Microelectron. Eng., 98, 472-476, 2012.

Zimmer, C. M., Asbeck, C., Lützenkirchen-Hecht, D., Glösekötter, P., and Kallis, K. T.: Backside illumination of an electronic photo ionization detector realized by UV transparent thin films, J. Nano Res., 25, 55-60, 2013.

Zimmer, C. M., Yoganathan, K., Giebel, F. J., Lützenkirchen-Hecht, D., Glösekötter, P., and Kallis, K. T.: Photoemission properties of $\mathrm{LaB}_{6}$ thin films for the use in PIDs, Proceedings of the 14th IEEE International Conference on Nanotechnology, Toronto, Canada, 18-21 August 2014, 877-881, 2014. 